\section{Validade da estrutura fatorial da escala de capital social utilizada na linha de base no ELSA-Brasil}

\author{
Factor structure validity of the social capital scale \\ used at baseline in the ELSA-Brasil study
}

\author{
Validez de la estructura factorial de la escala de \\ capital social, utilizada en la línea de \\ base del ELSA-Brasil
}

\section{Resumo}

Esta pesquisa tem por objetivo estudar a estrutura fatorial da versão brasileira da escala Resource Generator (RG), utilizando dados da linha de base do Estudo Longitudinal de Saúde do Adulto (ELSA-Brasil). Realizou-se validade cruzada com três subamostras aleatórias. A análise fatorial exploratória usando modelos de equação estrutural exploratória foi realizada nas duas primeiras subamostras para diagnóstico da estrutura fatorial e, na terceira, a análise fatorial confirmatória foi utilizada para corroborar o modelo definido pelas análises exploratórias. Com base nos 31 itens iniciais, o modelo de melhor ajuste contemplou 25 itens distribuídos em três dimensões. Todas apresentaram validade convergente (valores acima de 0,50 para a variância extraída) e precisão (valores acima de 0,70 para a confiabilidade composta) satisfatórias. Todas as correlações fatoriais foram abaixo de 0,85 , indicando plena validade fatorial discriminante. A escala RG apresenta propriedades psicométricas aceitáveis e pode ser utilizada em populações com características semelhantes.

Inquéritos e Questionários; Capital Social; Estudos de Validação; Análise Fatorial
Ester Paiva Souto 1

Ana Glória Godoi Vasconcelos 1

Dora Chor 1

Michael E. Reichenheim 2

Rosane Härter Griep ${ }^{3}$

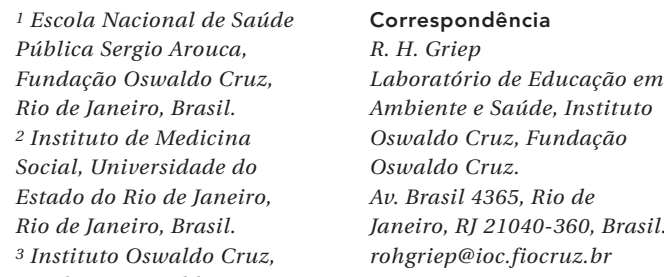

1 Escola Nacional de Saúde Pública Sergio Arouca Fundação Oswaldo Cruz Rio de Janeiro, Brasil. 2 Instituto de Medicina Social, Universidade do Estado do Rio de Janeiro, Rio de Janeiro, Brasil. 3 Instituto Oswaldo Cruz Fundação Oswaldo Cruz, Rio de Janeiro, Brasil. 


\section{Introdução}

Capital social se refere à estrutura das redes sociais 1 e aos recursos nelas inseridos 2 . Aspectos como a confiança social, a reciprocidade e as normas que constituem as redes de relações são centrais na formulação de seu constructo 3,4 . A associação entre baixos níveis de capital social e diversos desfechos de saúde, tais como, transtornos mentais comuns ${ }^{5}$, depressão ${ }^{6,7,8}$, condições de saúde autorreferidas 9,10,11 e comportamento suicida 12,13 já foi demonstrada, além de associações com comportamentos de saúde, tais como sedentarismo 14 e tabagismo 15. Por outro lado, níveis elevados de capital social foram relacionados à diminuição da obesidade 16 e ao controle do diabetes 17 .

Com a crescente produção no campo temático do capital social, estudos sobre suas associações com desfechos de saúde têm se mostrado cada vez mais frequentes. No entanto, os resultados devem ser interpretados com cautela, pois há ainda um amplo debate acerca da forma mais adequada de análise dos efeitos do capital social, considerando sua dimensão coletiva em oposição à percepção individual ${ }^{18}$. No nível coletivo, estuda sistemas horizontais de participação cívica, cujas características centrais envolvem confiança, cooperação e reciprocidade, o que amplia demasiadamente o seu escopo de análise 18. Medidas usuais incluem coletar dados individuais e agrupá-los no nível da vizinhança, da cidade ou até mesmo de um país. O nível individual está frequentemente associado à análise de redes sociais, na qual a investigação prioriza a percepção subjetiva de aspectos relacionados ao volume (número de pessoas que compõem a rede), à estrutura (análise da densidade da rede) e à sua diversidade (tipos de laços, contato com pessoas de diferentes idades, sexo e escolaridade) 19,20 .

Um recente artigo de revisão 21 apontou que, dentre os instrumentos que avaliaram o capital social em relação à saúde em países de renda média ou baixa, a maioria avaliou no nível individual. Os instrumentos apresentam grande variedade no número de itens e dimensões avaliados e poucos apresentaram suas propriedades psicométricas. Ainda não há consenso sobre o melhor instrumento que avalie de forma abrangente a influência dos recursos sociais sobre a saúde de grupos populacionais. Dentre os instrumentos disponíveis, o Resource Generator (RG) apresenta situações do cotidiano para discriminar o acesso a diversos tipos de recursos, é considerado um instrumento de aplicação fácil, objetivo e com interpretações mais claras sobre o capital social de determinada população 22 .
O RG é novo no contexto internacional e poucos estudos de adaptação transcultural foram realizados 5,23,24,25. Adaptações locais da escala e avaliação da pertinência são recomendadas, uma vez que o capital social é um constructo dependente de normas culturais que podem variar entre diferentes contextos 22. Corroborando essa afirmação, a escala sofreu modificações em todos os países em relação ao número e conteúdo dos itens, e até mesmo à sua dimensionalidade.

Elaborado na Holanda, o instrumento tinha incialmente 33 itens. Sua validade dimensional foi avaliada por meio do método Mokken de escalabilidade (modelo matemático não paramétrico da teoria de resposta ao item que possibilita avaliar o quanto a escala construída se afasta da escala determinística de Guttman), e apenas 17 itens foram mantidos nas análises. Essa versão apresentou boa escalabilidade e foi identificada usando-se o coeficiente de Loevinger $(0,4<\mathrm{H}<$ $0,49)$. A consistência interna mostrou ser de moderada a muito boa $(0,54<$ Rho $<0,86)$ em suas quatro dimensões 22

Webber \& Huxley 5 foram os primeiros a adaptar o RG para uso no Reino Unido. Ainda que mantendo a estrutura configural do instrumento original, 12 itens foram excluídos dos 33 iniciais, seis foram adicionados e os demais foram reformulados. Esse processo resultou em uma versão de 27 itens. Sua validade, também aferida pelo método Mokken, foi considerada adequada $(0,37<\mathrm{H}<0,54)$ e a consistência interna variou entre boa e muito boa $(0,6<$ Rho $<0,83)$. Outra versão modificada do RG foi aplicada na Bélgica e está validada em alemão. Uma análise de componentes principais aplicada ao instrumento de 33 itens identificou quatro fatores. No entanto, apenas dois apresentaram confiabilidade adequada quando avaliados por meio do coeficiente alfa de Cronbach 23 .

Outras versões reduzidas e modificadas também foram elaboradas. Nos Estados Unidos, Grinstein-Weiss et al. 24 estudaram a dimensionalidade da escala valendo-se de uma análise fatorial exploratória, encontrando uma estrutura unidimensional com oito itens. Já Häuberer 25, na República Tcheca, propôs 12 itens distribuídos em três dimensões utilizando análise fatorial exploratória e análise fatorial confirmatória.

O RG foi incluído como um dos determinantes sociais de saúde na linha de base do Estudo Longitudinal de Saúde do Adulto (ELSA-Brasil). As etapas iniciais do processo de adaptação transcultural estão detalhadas em outra publicação 26. O instrumento aplicado por meio de entrevistas face a face apresentou estimativas aceitáveis de confiabilidade teste-reteste. $\mathrm{O}$ objetivo do presente artigo é complementar o estudo das 
propriedades psicométricas por meio da avaliação da estrutura fatorial da versão brasileira da escala RG, utilizando dados da linha de base do ELSA-Brasil.

\section{Métodos}

\section{Desenho de estudo, população e coleta e dados}

O ELSA-Brasil é um estudo de coorte com 15.105 servidores públicos ativos e aposentados com idades entre 35 e 74 anos, de seis instituições de ensino e pesquisa brasileiras. A coleta de dados da linha de base foi realizada no período de 2008 2010. Após a assinatura do termo de consentimento, os questionários foram aplicados nos Centros de Investigação ELSA por entrevistadores treinados e certificados. Outras publicações detalham os objetivos do estudo e seu delineamento 27,28 , bem como a descrição de medidas de garantia e controle de qualidade de todo o processo de coleta dos dados 29 . Para as análises do presente artigo foram excluídos os participantes que deixaram de responder a algum dos itens de capital social $(n=54)$, resultando em uma amostra com 15.051 indivíduos $(99,6 \%)$.

\section{O instrumento Resource Generator}

O RG é um instrumento que busca captar a percepção individual de capital social representado por situações práticas para avaliar o acesso aos diversos tipos de recursos 22. O instrumento foi adaptado ao contexto brasileiro seguindo as recomendações de adaptações culturais locais 26 . Assim, a versão original de 33 itens resultou em 31 (dois itens foram incluídos e quatro foram excluídos), com categorias de respostas dicótomas ( $\operatorname{sim} /$ não). O instrumento se propõe a cobrir quatro dimensões da vida, consideradas essenciais ao capital social: (1) prestígio e educação (conhecer alguém que leia livros de literatura); (2) habilidades políticas e financeiras (conhecer alguém que possa discutir sobre candidatos nas eleições); (3) habilidades pessoais (conhecer alguém que fale uma língua estrangeira); e (4) apoio pessoal (conhecer alguém que possa tomar conta das crianças) 22 .

\section{Análise de dados}

Conforme descrito na introdução, embora alguns estudos tenham avaliado a estrutura fatorial do instrumento, em todos eles houve adaptações locais e modificações importantes desta estrutura, tanto no que se refere ao número de itens quanto ao número de dimensões subjacentes 5,23,24,25. Além disso, a definição da pertinência de cada um dos 33 itens originais nas suas respectivas dimensões não foi apresentada 22. Esses aspectos, aliados ao tamanho amostral do ELSA-Brasil, nortearam as opções pelas etapas da avaliação da estrutura fatorial que incluíram análises fatoriais exploratórias e confirmatórias, além de validade cruzada.

O banco de dados foi repartido em três subamostras aleatórias $(\mathrm{n} 1=6.020 ; \mathrm{n} 2=4.515$ e n3 = 4.516), analisadas de maneira independente em duas etapas, conforme indicado na Figura 1. Na primeira etapa, duas subamostras serviram para diagnósticos da estrutura fatorial por meio de sucessivas análises fatoriais exploratórias usando modelos de equação estrutural exploratórios (MEEE) 30. A análise fatorial exploratória (AFE) se aplica ao diagnóstico da estrutura fatorial, sem partir de pressupostos sobre o número de fatores e o conjunto de itens que os definem. A modelagem de equações estruturais permite investigar simultaneamente diversas relações de dependência entre as variáveis, baseando-se em técnicas de análise multivariada 31,32 .

Começando com o instrumento "pleno" de 31 itens, a cada análise o comportamento das cargas dos itens nas dimensões foi avaliado, adotando-se como critérios de exclusão cargas abaixo de 0,35 ou cargas cruzadas com diferença $<0,2$ entre as duas maiores cargas. Todos os itens que atendessem a pelo menos um desses critérios simultaneamente nas duas primeiras subamostras seriam retirados.

A análise fatorial confirmatória (AFC) é utilizada quando se deseja corroborar uma estrutura dimensional predefinida e desta forma, na segunda etapa, o modelo definido na primeira foi testado por meio de uma AFC na terceira subamostra. Além dos componentes métricos habituais (cargas fatoriais e respectivos resíduos), as análises também incluíram a confiabilidade composta (consistência interna) 33 e as validades fatoriais convergente e discriminante. A validade convergente refere-se ao grau em que indicadores de um constructo específico compartilham uma elevada proporção de sua variância comum. Se o valor for alto, significa dizer que o instrumento está medindo o que pretende medir 31,33. A validade discriminante refere-se à capacidade do constructo de ser verdadeiramente distinto de outros.

A confiabilidade composta (CC) e a variância média extraída (VME), nas quais se esteiam as validades fatoriais convergente e discriminante, foram estimadas para cada fator como funções das cargas fatoriais padronizadas dos itens componentes e dos respectivos erros de mensura- 


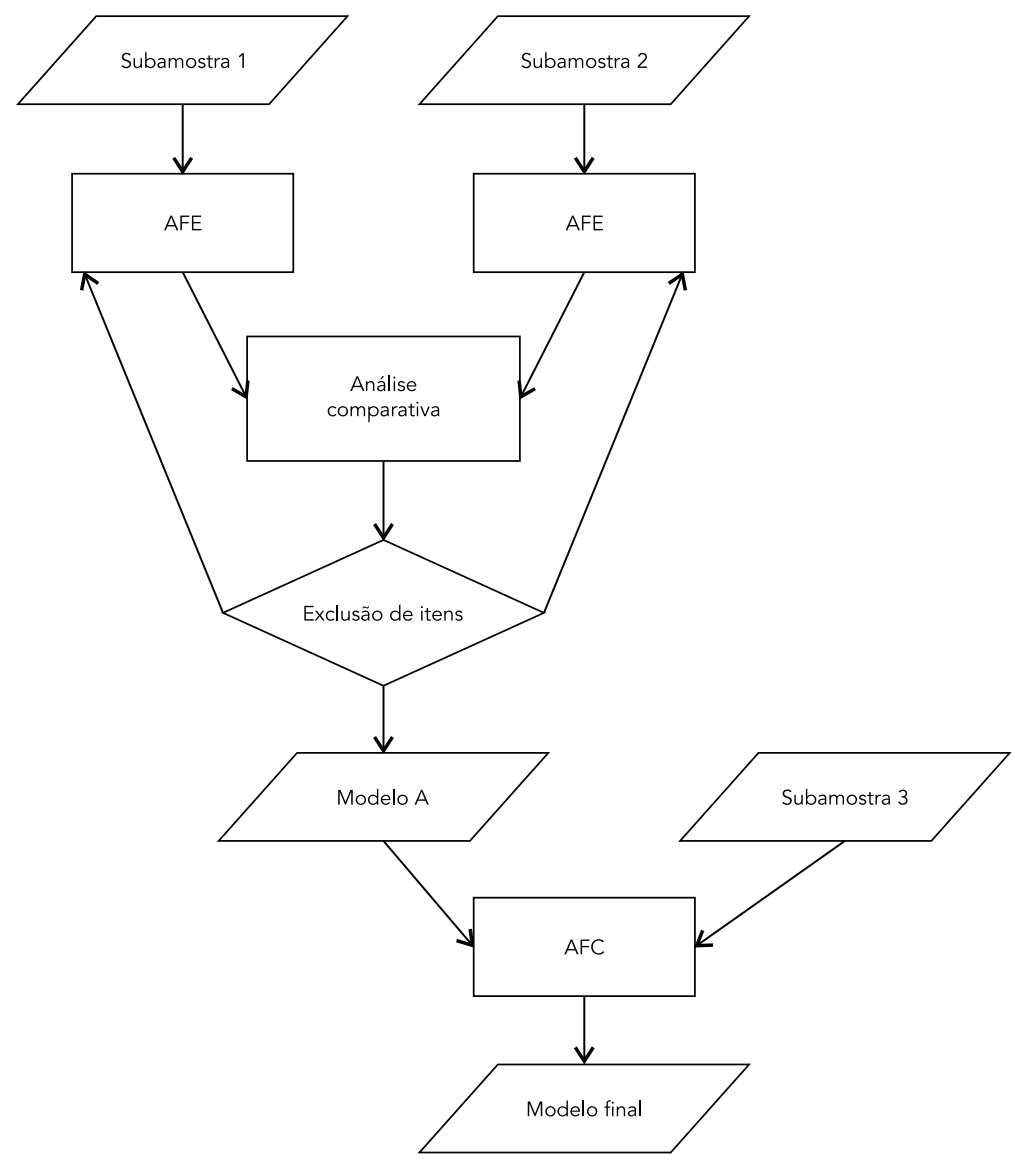

AFC: análise fatorial confirmatória; AFE: análise fatorial exploratória.

ção. A confiabilidade composta avalia se os itens apresentam consistência interna, e para cada fator sua estimativa varia de 0 a 1 e valores $\geq 0,70$ sugerem consistência interna satisfatória 31,32. A estimativa da VME também varia de 0 a 1 . Um fator apresenta boa validade convergente se $P_{v m e\left(f_{j}\right)}$ $\geq 0,50$, o que indica que pelo menos $50 \%$ da variância em uma medida é devido ao traço latente hipotetizado 32 . A avaliação da validade fatorial discriminante se baseou no contraste entre a raiz quadrada da VME do fator $\sqrt{\rho_{\text {vme }\left(f_{j}\right)}}$ e as correlações com outros fatores do sistema $\Phi_{\left(f_{j} \leftrightarrow f_{* j}\right)}$. Levando-se em conta que aqui também os valores variam de 0 a 1 , considerou-se a validade fatorial discriminante como sustentável se, por fator, exaustivamente $\sqrt{\rho_{v m e\left(f_{j}\right)}}>\Phi_{\left(f_{j} \leftrightarrow f_{* j}\right)} 34$. Para sustentar uma validade fatorial discriminante utilizou-se adicionalmente um critério largamente recomendado na literatura afim que demarca $\Phi_{\left(f_{j}\right)}<0,8531$.

Todas as análises foram conduzidas no Mplus 7.1 (Muthén \& Muthén, Los Angeles, Estados Unidos). Para os procedimentos de MEEE e AFC, utilizou-se o estimador de mínimos quadrados ponderados robusto (weighted least squares means and variance - WLSMV), apropriado para variáveis (itens) dicótomos ou policótomos, conforme as características dos itens do instrumento RG. Ajustes de modelo foram avaliados pelo índice de ajuste comparativo (comparative fit index - CFI), pelo índice de Tucker-Lewis (Tucker-Lewis index - TLI) e pela raiz do erro médio quadrático de aproximação (root mean square error of aproximation - RMSEA). Os dois primeiros avaliam o ajuste incremental do modelo comparado com um modelo nulo. Ambos variam de 0 a 1 e valo- 
res acima de 0,95 indicam ajuste adequado 31 . O RMSEA incorpora uma função de penalidade para lidar com a pouca parcimônia expressa pelos graus de liberdade do modelo 35 . Valores $<0,06$ sugerem um bom ajuste, e os valores $>0,10$ indicam ajuste ruim e a rejeição do modelo 31 .

\section{Resultados}

De maneira geral, a população do estudo tinha em média 52 anos (DP $=9,0$ ). Pouco mais da metade $(54,4 \%)$ eram mulheres; $12,7 \%$ tinham apenas o Ensino Fundamental; e 52,6\% tinham o Ensino Superior ou Pós-graduação. A renda domiciliar média per capita dos participantes era de 4,2 (DP = 3,5) salários mínimos, conforme valor vigente em 2008 (R\$ 415,00); 80,1\% eram funcionários ativos das instituições públicas envolvidas no estudo. Não foram observadas variações significativas dessas características nas três subamostras utilizadas (dados não apresentados).

Diversos itens apresentaram altas frequências de respostas afirmativas. O item 2 - "conhecer alguém que possui um carro" - apresentou frequência extremamente alta $(99,9 \%)$, sendo excluído das análises principais por carecer de poder discriminativo. Dentre os demais, os itens mais frequentes foram conhecer alguém: "que tenha se formado no segundo grau" (99\%), "que possa trabalhar com um computador" $(99,4 \%)$, "para fazer compras se estiver doente" (97,3\%), "que possa dar boas referências sobre emprego" $(97,2 \%)$ e "para se abrigar em casa por uma semana" (96,9\%). Outros itens referentes às atividades do dia a dia (por exemplo, os itens 21, 22, 29 e 30) foram endossados por mais de $90 \%$ dos participantes. As frequências mais baixas de endosso foram encontradas em itens que, de maneira mais ampla, conectam os indivíduos com outros níveis da hierarquia social ou outras esferas da vida, como "conhecer alguém que possa conseguir um emprego" (56,7\%), "ter conexões com a mídia" (59,3\%) e "conhecer um ativista político" $(66,3 \%)$.

\section{Análise de fatores exploratória}

Baseada na adequação dos ajustes de modelo e na distribuição de cargas que propiciavam a melhor defesa teórica das dimensões, a exploração inicial da estrutura fatorial via MEEE identificou três fatores nas subamostras avaliadas (Tabela 1). Em seguida, procedeu-se à inspeção das cargas fatoriais de acordo com os critérios de exclusão estabelecidos (magnitude de carga $<0,35$ e/ou carga cruzada com diferença abaixo de 0,2 entre as duas maiores cargas, simultaneamente em ambas as subamostras). As cargas dos cinco itens que atenderam a esses critérios estão destacadas em negrito na Tabela 1 e os detalhes na Tabela 2. À retirada desses itens, a segunda rodada de MEEEs não revelou outros indicadores passíveis de exclusão (Tabela 3). Os índices de ajuste do novo modelo mostraram-se adequados nas duas subamostras. As correlações fatoriais aumentaram ligeiramente em relação às soluções anteriores (Tabela 1), mas, consistentemente, as maiores continuaram sendo entre os fatores $1 \mathrm{e}$ $3\left(\Phi_{\left(f_{1} \leftrightarrow f_{3}\right)}=0,629\right.$ na primeira e $\Phi_{\left(f_{1} \leftrightarrow f_{3}\right)}=0,714$ na segunda subamostras).

\section{Análise de fatores confirmatória}

Identificado um modelo a partir das explorações inicias, a análise confirmatória realizada na terceira subamostra mostrou que, à exceção dos itens 1 ("consertar um carro, uma bicicleta etc.") e 12 ("trabalhe na prefeitura ou governo do estado"), todos os outros tinham cargas fatoriais acima de 0,6 (Tabela 4). Os índices de ajuste foram adequados. As estimativas de VME e de CC superaram os pontos de corte de 0,50 e 0,70 , respectivamente, indicando haver validade convergente e aceitável consistência interna. Os valores da raiz quadrada das VMEs dos fatores $2 \mathrm{e}$ $3\left(\sqrt{\rho_{\text {vme }(f 2)}=0,742}\right.$ e $\sqrt{\rho_{\text {vme }(f 3)}=0,755}$, respectivamente) foram um pouco menores do que a correlação entre eles $\left(\Phi_{\left(f_{2} \leftrightarrow f_{3}\right)}=0,766\right)$, apontando para uma situação limítrofe. No entanto, essa correlação foi inferior ao ponto de corte adotado de $<0,85$, o que admitiria a existência de validade fatorial discriminante. Nessa perspectiva, os resultados do processo sequencial implementado nas três subamostras sugeriram uma estrutura com 25 itens distribuídos em três dimensões, nomeadas como habilidades simples, prestígio, e educação e apoio social.

\section{Discussão}

A estrutura fatorial de melhor ajuste para a versão brasileira da escala RG tem cargas fatoriais adequadas para quase todos os itens e bons índices de ajuste. Embora a validade discriminante dos três fatores possa ser considerada adequada, um destes (habilidades simples) acabou constituído por apenas dois itens no final do processo. Esses achados são discutidos nos parágrafos que seguem.

O constructo capital social é fortemente dependente de cultura e contexto. Assim, adotou-se a recomendação do autor 22 de adaptar a escala original para, no processo, identificar a pertinência dos itens componentes no contexto 
Tabela 1

Primeira análise exploratória da escala Resource Generator (RG) usando modelos de equação estrutural exploratória (MEEE): cargas padronizadas, erros de mensuração e estimativas de ajuste geral do modelo nas subamostras 1 e 2. ELSA-Brasil, 2008-2010.

\begin{tabular}{|c|c|c|c|c|c|c|c|c|}
\hline \multirow[t]{2}{*}{ Itens } & \multicolumn{4}{|c|}{ AFE 1 (subamostra 1) } & \multicolumn{4}{|c|}{ AFE 1 (subamostra 2) } \\
\hline & $\lambda \mathrm{i} 1$ & $\lambda \mathrm{i} 2$ & $\lambda i 3$ & $\delta \mathbf{i}$ & $\lambda \mathrm{i} 1$ & $\lambda \mathrm{i} 2$ & $\lambda \mathrm{i} 3$ & $\delta \mathbf{i}$ \\
\hline 1. Consertar um carro, uma bicicleta etc. & $-0,120$ & 0,562 & 0,118 & 0,668 & $-0,059$ & 0,434 & 0,113 & 0,792 \\
\hline 3. Habilidade consertar equipamentos doméstico & 0,175 & 0,421 & 0,143 & 0,655 & 0,191 & 0,414 & 0,104 & 0,649 \\
\hline 4. Fale uma língua estrangeira & 0,896 & $-0,052$ & $-0,097$ & 0,312 & 0,935 & 0,005 & $-0,146$ & 0,272 \\
\hline 5. Trabalhar com um computador & 0,699 & 0,149 & $-0,062$ & 0,479 & 0,524 & 0,250 & 0,004 & 0,536 \\
\hline 6. Tocar algum instrumento musical & 0,526 & 0,284 & $-0,013$ & 0,561 & 0,542 & 0,284 & $-0,080$ & 0,544 \\
\hline 7. Leia livros de literatura & 0,843 & $-0,035$ & $-0,007$ & 0,313 & 0,783 & 0,048 & $-0,028$ & 0,376 \\
\hline 8. Formou-se no segundo grau & 0,677 & 0,010 & $-0,016$ & 0,550 & 0,614 & $-0,004$ & 0,072 & 0,565 \\
\hline 9. Leia revistas profissionais & 0,701 & 0,028 & 0,097 & 0,403 & 0,670 & 0,178 & $-0,015$ & 0,421 \\
\hline 10. Ativista de partido político & 0,528 & 0,462 & $-0,154$ & 0,479 & 0,399 & 0,454 & $-0,075$ & 0,520 \\
\hline 11. Tenha mais de $R \$ 2.000,00$ na poupança & 0,762 & $-0,092$ & 0,135 & 0,323 & 0,860 & $-0,133$ & 0,084 & 0,262 \\
\hline 12. Trabalhe na prefeitura ou governo do estado & 0,364 & 0,362 & $-0,027$ & 0,672 & 0,220 & 0,462 & $-0,006$ & 0,646 \\
\hline 13. Ganhe mais de $\mathrm{R} \$ 2.000,00$ por mês & 0,813 & $-0,013$ & 0,027 & 0,319 & 0,914 & $-0,118$ & 0,011 & 0,242 \\
\hline 14. Tenha casa de campo ou praia & 0,610 & 0,151 & 0,053 & 0,502 & 0,603 & 0,239 & $-0,013$ & 0,454 \\
\hline 15. Contrate pessoas para trabalhar & 0,661 & 0,149 & 0,078 & 0,405 & 0,575 & 0,210 & 0,093 & 0,420 \\
\hline 16. Conheça sobre leis e regulamentos do governo & 0,620 & 0,300 & 0,019 & 0,392 & 0,491 & 0,368 & 0,033 & 0,422 \\
\hline 17. Contatos com imprensa, rádio ou TV & 0,531 & 0,421 & 0,006 & 0,397 & 0,444 & 0,465 & 0,006 & 0,385 \\
\hline 18. Conheça bem assuntos financeiros & 0,524 & 0,318 & 0,110 & 0,417 & 0,434 & 0,386 & 0,073 & 0,438 \\
\hline 19. Arranjar emprego para família & 0,231 & 0,393 & 0,359 & 0,411 & 0,112 & 0,428 & 0,410 & 0,405 \\
\hline 20. Dar conselhos a conflitos no trabalho & 0,009 & 0,225 & 0,639 & 0,433 & $-0,026$ & 0,352 & 0,553 & 0,453 \\
\hline 21. Ajudar a fazer uma mudança de casa & $-0,022$ & 0,105 & 0,677 & 0,499 & $-0,100$ & 0,159 & 0,708 & 0,484 \\
\hline 22. Ajudar em pequenas tarefas domésticas & 0,121 & $-0,037$ & 0,688 & 0,432 & 0,135 & 0,035 & 0,643 & 0,437 \\
\hline 23. Possa fazer compra se estiver doente & 0,000 & $-0,123$ & 0,779 & 0,445 & $-0,005$ & $-0,078$ & 0,794 & 0,414 \\
\hline 24. Emprestar um dinheiro se precisar & 0,335 & $-0,016$ & 0,510 & 0,435 & 0,337 & $-0,039$ & 0,550 & 0,377 \\
\hline 25. Abrigar em casa por uma semana & 0,265 & $-0,193$ & 0,664 & 0,366 & 0,273 & $-0,122$ & 0,590 & 0,445 \\
\hline 26. Conselhos sobre conflitos na família & $-0,109$ & 0,140 & 0,828 & 0,318 & 0,010 & 0,131 & 0,727 & 0,374 \\
\hline 27. Discutir candidato nas eleições & 0,457 & 0,161 & 0,270 & 0,471 & 0,341 & 0,250 & 0,313 & 0,451 \\
\hline 28. Dar boas referências sobre emprego & 0,239 & 0,044 & 0,532 & 0,485 & 0,329 & 0,139 & 0,377 & 0,492 \\
\hline 29. Tomar conta das crianças & 0,090 & $-0,016$ & 0,703 & 0,432 & 0,076 & $-0,014$ & 0,715 & 0,423 \\
\hline 30. Conversar assuntos importantes & 0,206 & 0,032 & 0,678 & 0,313 & 0,184 & 0,030 & 0,650 & 0,372 \\
\hline 31. Facilitar internação ou exame & 0,162 & 0,242 & 0,367 & 0,624 & 0,049 & 0,317 & 0,407 & 0,596 \\
\hline$\Phi_{(f 1 \Leftrightarrow f 2)}$ & \multicolumn{4}{|c|}{0,310} & \multicolumn{4}{|c|}{0,475} \\
\hline$\Phi_{(f 1 \Leftrightarrow f 3)}$ & \multicolumn{4}{|c|}{0,589} & \multicolumn{4}{|c|}{0,629} \\
\hline$\Phi_{(f 2 \Leftrightarrow f 3)}$ & \multicolumn{4}{|c|}{0,351} & \multicolumn{4}{|c|}{0,367} \\
\hline $\mathrm{CFI} / \mathrm{TLI}$ & \multicolumn{4}{|c|}{$0,980 / 0,976$} & \multicolumn{4}{|c|}{$0,982 / 0,978$} \\
\hline RMSEA * & \multicolumn{4}{|c|}{$0,025(0,023-0,026)$} & \multicolumn{4}{|c|}{$0,023(0,022-0,025)$} \\
\hline
\end{tabular}

AFE: análise fatorial exploratória; CFI: comparative fit index; RMSEA: root mean square error of aproximation; TLI: Tucker-Lewis index.

* Entre parênteses: intervalos de $90 \%$ de confiança.

Nota: a maior carga em cada item está sombreada. 
Critérios de exclusão utilizados nos itens retirados após a primeira análise exploratória nas subamostras 1 e 2 .

\begin{tabular}{|c|c|c|}
\hline \multirow[t]{2}{*}{ Itens excluídos } & \multicolumn{2}{|c|}{ Critérios } \\
\hline & AFE1 (subamostra 1) & AFE1 (subamostra 2) \\
\hline 10. Ativista de partido político & $\neq$ de 0,07 entre as duas maiores cargas & $\neq$ de 0,05 entre as duas maiores cargas \\
\hline 17. Contatos com imprensa, rádio ou TV & $\neq$ de 0,11 entre as duas maiores cargas & $\neq$ de 0,02 entre as duas maiores cargas \\
\hline 19. Arranjar emprego para família & $\neq$ de 0,03 entre as duas maiores cargas & $\neq$ de 0,02 entre as duas maiores cargas \\
\hline 27. Discutir candidato em eleições & $\neq$ de 0,19 entre as duas maiores cargas & Carga principal com valor de 0,31 \\
\hline 31. Facilitar internação ou exame & $\neq$ de 0,13 entre as duas maiores cargas & $\neq$ de 0,09 entre as duas maiores cargas \\
\hline
\end{tabular}

AFE: análise fatorial exploratória

brasileiro. Não surpreende que a dimensionalidade encontrada no contexto brasileiro tenha sido diferente da encontrada na escala original, assim como ocorreu em estudos pregressos. Três desses estudos relataram um número de dimensões semelhante ao da adaptação brasileira, mas há grandes diferenças quanto à quantidade e ao conteúdo semântico dos itens 5,23. Outros dois trabalhos propuseram versões uni e bidimensional, respectivamente ${ }^{25,24}$. A diversidade de metodologias de análise usadas nesses trabalhos pode ter contribuído para a discrepância das estruturas identificadas. Cabe notar que essa diferença metodológica no processo de avaliação, aliada à variabilidade de conteúdo dos instrumentos e do número de itens, dificulta a comparação entre os diferentes estudos.

O modelo final apresentado corrobora a propriedade multidimensional da escala 22 . No entanto, de maneira diferente daquela encontrada na versão original, os itens da dimensão habilidades políticas e financeiras ("conheça sobre leis e regulamentos do governo" e "conheça bem assuntos financeiros") ficaram agrupados na dimensão prestígio e educação, da mesma forma que alguns itens que, na escala original, pertencem à dimensão habilidades pessoais ("leia revistas profissionais", "possa trabalhar com um computador" e "possa falar uma língua estrangeira"). Portanto, no contexto brasileiro, esses itens parecem estar representando recursos providos por pessoas que apresentam maior escolaridade e que ocupam posições superiores na hierarquia social. Além disso, a dimensão nomeada apoio social contemplou apenas quatro itens da mesma dimensão no estudo original.

No presente estudo, a dimensão habilidades simples apresentou apenas dois itens, que na escala original foram excluídos após as análises estatísticas, sendo que um deles ("consertar um carro, uma bicicleta etc.”) apresentou carga re- lativamente baixa $(0,549)$. Claramente, essa dimensão não atendeu à exigência mínima de três indicadores por fator e que, segundo alguns autores 36,37 , permitiria um mapeamento mais adequado do crescente de intensidade do conteúdo dimensional. Esse resultado limita, portanto, o uso da dimensão em estudos posteriores. É possível que essa dimensão possa ter um significado diferenciado em grupos socioeconomicamente desfavorecidos, não incluídos na população do ELSA-Brasil. Portanto, avaliações da sua aplicabilidade em populações com características diferentes poderiam complementar análises da pertinência dessa dimensão do instrumento.

Considerando-se que todas as correlações entre fatores foram menores que 0,85, é possível sugerir uma aceitável validade fatorial discriminante na estrutura fatorial do modelo final. No entanto, essa qualificação merece prudência, uma vez que o contraste da correlação entre fatores representando prestígio e educação (f2) e apoio social $(f 3)$ com as respectivas raízes quadradas das VMEs aponta para uma situação limítrofe. Quando dois ou mais fatores têm uma forte correlação, é possível conjeturar a existência de um fator de ordem superior responsável pela variância por eles partilhada. Dessa forma, há bases teóricas para supor a existência de tal fator de ordem superior. Investigações adicionais permitirão testar a adequação dessa hipótese.

Alguns itens apresentaram cargas baixas ou cruzadas nas avaliações exploratórias sequenciais: item 27 ("discutir candidato nas eleições"), item 10 (“ativista de partido político"), item 17 ("contatos com imprensa, rádio ou TV") e item 19 (“arranjar emprego para família”). É interessante notar que esses três últimos foram os menos endossados, cabendo, pois, especular que tenham sido mal interpretados pelos participantes. Estudos qualitativos adicionais poderão contribuir para uma melhor compreensão sobre o que 
Tabela 3

Segunda análise exploratória da escala Resource Generator (RG) usando modelos de equação estrutural exploratória (MEEE): cargas padronizadas, erros de mensuração e estimativas de ajuste geral do modelo nas subamostras 1 e 2. ELSA-Brasil, 2008-2010.

\begin{tabular}{|c|c|c|c|c|c|c|c|c|}
\hline \multirow[t]{2}{*}{ Itens } & \multicolumn{4}{|c|}{ AFE 2 (subamostra 1) } & \multicolumn{4}{|c|}{ AFE 2 (subamostra 2) } \\
\hline & $\lambda \mathrm{i} 1$ & $\lambda \mathrm{i} 2$ & $\lambda i 3$ & $\delta \mathbf{i}$ & $\lambda \mathrm{i} 1$ & $\lambda \mathrm{i} 2$ & $\lambda \mathrm{i} 3$ & $\delta \mathbf{i}$ \\
\hline 1. Consertar um carro, uma bicicleta etc. & 0,002 & 0,726 & 0,020 & 0,462 & 0,395 & 0,551 & 0,017 & 0,605 \\
\hline 3. Habilidade consertar equipamento doméstico & 0,313 & 0,567 & 0,016 & 0,498 & 0,627 & 0,481 & 0,012 & 0,468 \\
\hline 4. Fale uma língua estrangeira & 0,903 & $-0,042$ & $-0,134$ & 0,326 & 0,882 & $-0,116$ & $-0,104$ & 0,294 \\
\hline 5. Trabalhar com um computador & 0,758 & 0,236 & $-0,143$ & 0,432 & 0,761 & 0,229 & $-0,040$ & 0,470 \\
\hline 6. Tocar algum instrumento musical & 0,573 & 0,245 & $-0,018$ & 0,570 & 0,740 & 0,151 & $-0,071$ & 0,537 \\
\hline 7. Leia livros de literatura & 0,870 & 0,011 & $-0,083$ & 0,321 & 0,774 & $-0,077$ & $-0,003$ & 0,377 \\
\hline 8. Formou-se no segundo grau & 0,695 & 0,009 & $-0,046$ & 0,553 & 0,620 & 0,009 & 0,056 & 0,565 \\
\hline 9. Leia revistas profissionais & 0,756 & 0,071 & 0,008 & 0,394 & 0,772 & $-0,004$ & $-0,015$ & 0,419 \\
\hline 11. Tenha mais de $R \$ 2.000,00$ na poupança & 0,778 & $-0,140$ & 0,107 & 0,314 & 0,649 & $-0,305$ & 0,145 & 0,259 \\
\hline 12. Trabalhe na prefeitura ou governo do estado & 0,431 & 0,229 & $-0,007$ & 0,726 & 0,528 & 0,189 & 0,002 & 0,718 \\
\hline 13. Ganhe mais de $R \$ 2.000,00$ por mês & 0,839 & $-0,058$ & 0,006 & 0,306 & 0,719 & $-0,298$ & 0,071 & 0,241 \\
\hline 14. Tenha casa de campo ou praia & 0,653 & 0,093 & 0,042 & 0,502 & 0,769 & 0,063 & $-0,024$ & 0,447 \\
\hline 15. Contrate pessoas para trabalhar & 0,725 & 0,088 & 0,034 & 0,407 & 0,700 & $-0,010$ & 0,080 & 0,421 \\
\hline 16. Conheça sobre leis e regulamentos do governo & 0,696 & 0,165 & 0,012 & 0,431 & 0,743 & 0,092 & 0,016 & 0,445 \\
\hline 18. Conheça bem assuntos financeiros & 0,623 & 0,175 & 0,081 & 0,459 & 0,676 & 0,073 & 0,068 & 0,484 \\
\hline 20. Dar conselhos a conflitos no trabalho & 0,076 & 0,126 & 0,639 & 0,457 & 0,187 & 0,152 & 0,553 & 0,506 \\
\hline 21. Ajudar a fazer uma mudança de casa & 0,001 & 0,159 & 0,655 & 0,480 & $-0,052$ & 0,177 & 0,748 & 0,470 \\
\hline 22. Ajudar em pequenas tarefas domésticas & 0,108 & 0,042 & 0,674 & 0,423 & 0,059 & 0,071 & 0,718 & 0,421 \\
\hline 23. Possa fazer compra se estiver doente & $-0,051$ & $-0,044$ & 0,790 & 0,442 & $-0,220$ & 0,008 & 0,917 & 0,399 \\
\hline 24. Emprestar um dinheiro se precisar & 0,362 & $-0,077$ & 0,484 & 0,446 & 0,155 & $-0,174$ & 0,641 & 0,374 \\
\hline 25. Abrigar em casa por uma semana & 0,242 & $-0,121$ & 0,636 & 0,392 & 0,021 & $-0,163$ & 0,705 & 0,443 \\
\hline 26. Conselhos sobre conflitos na família & $-0,063$ & 0,103 & 0,832 & 0,308 & 0,003 & 0,056 & 0,797 & 0,362 \\
\hline 28. Dar boas referências sobre emprego & 0,254 & $-0,015$ & 0,537 & 0,486 & 0,329 & $-0,025$ & 0,429 & 0,503 \\
\hline 29. Tomar conta das crianças & 0,092 & 0,013 & 0,683 & 0,443 & $-0,079$ & $-0,019$ & 0,818 & 0,415 \\
\hline 30. Conversar assuntos importantes & 0,232 & 0,036 & 0,650 & 0,318 & 0,074 & $-0,025$ & 0,740 & 0,366 \\
\hline$\Phi_{(f 1 \Leftrightarrow f 2)}$ & \multicolumn{4}{|c|}{0,201} & \multicolumn{4}{|c|}{$-0,171$} \\
\hline$\Phi_{(f 1 \Leftrightarrow f 3)}$ & \multicolumn{4}{|c|}{0,618} & \multicolumn{4}{|c|}{0,714} \\
\hline$\Phi_{(f 2 \Leftrightarrow f 3)}$ & \multicolumn{4}{|c|}{0,305} & \multicolumn{4}{|c|}{$-0,042$} \\
\hline $\mathrm{CFI} / \mathrm{TLI}$ & \multicolumn{4}{|c|}{$0,983 / 0,977$} & \multicolumn{4}{|c|}{$0,985 / 0,980$} \\
\hline RMSEA * & \multicolumn{4}{|c|}{$0,023(0,022-0,025)$} & \multicolumn{4}{|c|}{$0,022(0,020-0,023)$} \\
\hline
\end{tabular}

AFE: análise fatorial exploratória; CFI: comparative fit index; RMSEA: root mean square error of aproximation; TLI: Tucker-Lewis index.

* Entre parênteses: intervalos de $90 \%$ de confiança.

Nota: a maior carga em cada item está sombreada.

se entende por esses tipos de recursos no contexto brasileiro.

O expressivo tamanho da amostra permitiu conferir precisão às estimativas, bem como a possibilidade de se avaliar a estabilidade da estrutura fatorial da escala em três subamostras por meio da validade cruzada. Ao contrário de múltiplas análises realizadas em um único ban- co de dados de tamanho limitado, o particionamento de um banco de grande porte permite verificar a replicabilidade de uma configuração fatorial ${ }^{38}$. Outro aspecto positivo diz respeito ao baixo porcentual de dados faltantes. Aliado ao uso de ferramentas metodológicas refinadas e adequadas a estudos de validação, este conjunto confere robustez às análises apresentadas. 
Tabela 4

Análise fatorial confirmatória (AFC) da escala Resource Generator (RG): cargas padronizadas, erros de mensuração e estimativas de ajuste geral do modelo na subamostra 3. ELSA-Brasil, 2008-2010.

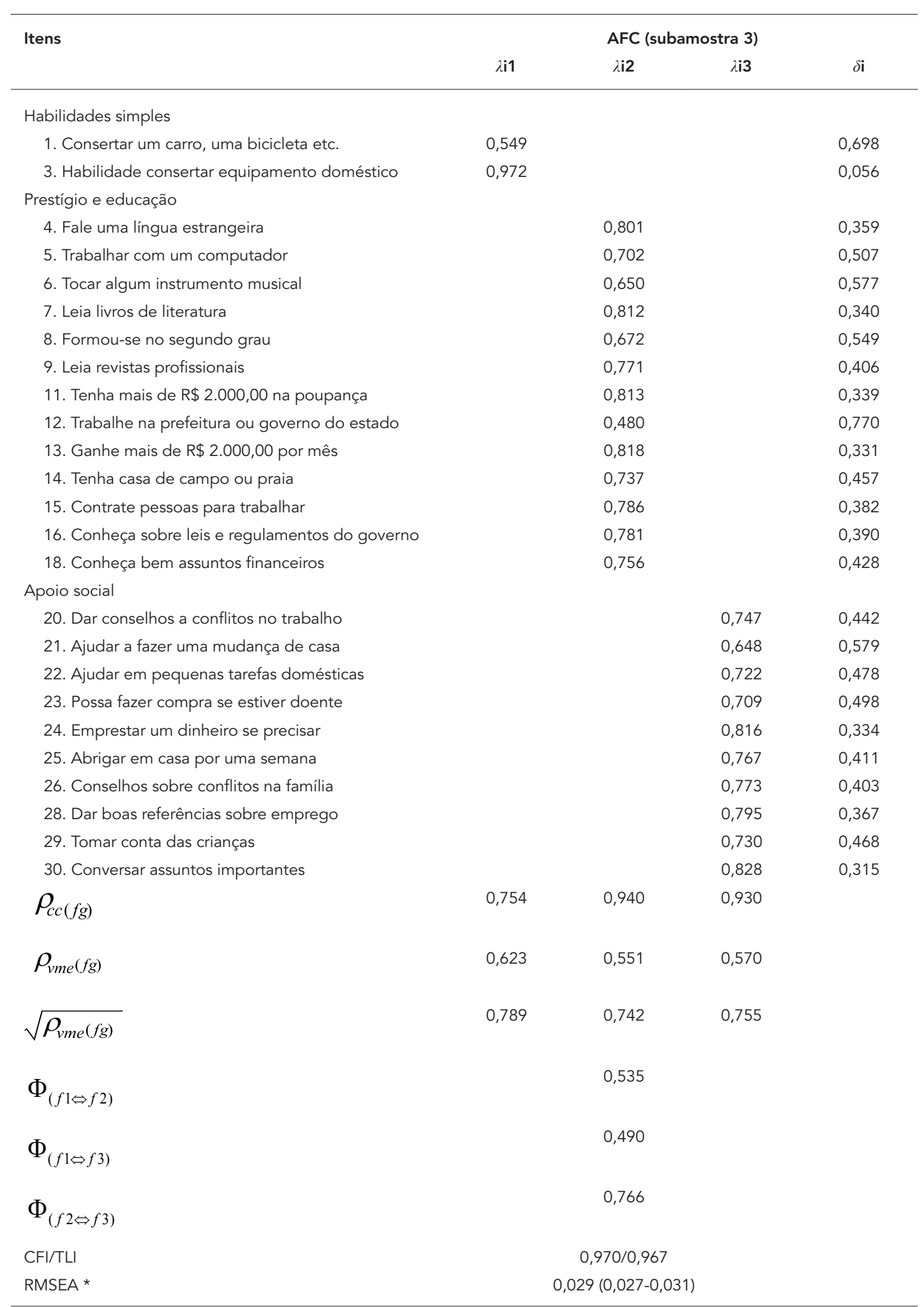

CFI: comparative fit index; RMSEA: root mean square error of aproximation; TLI: Tucker-Lewis index.

* Entre parênteses: intervalos de $90 \%$ de confiança. 
No lado negativo, destaca-se a alta frequência de endossos da maioria dos itens. Esse problema já havia sido apontado no estudo de confiabilidade teste-reteste no âmbito do ELSA-Brasil 26 e até mesmo em trabalhos anteriores 20 . No estudo holandês de concepção do instrumento, por exemplo, essa característica foi entendida como conferindo pouca especificidade aos itens e, por extensão, como uma limitação da escala em si 20. De fato, o grau de endosso foi ainda maior na população em estudo. É possível que as diferenças entre as populações estudadas expliquem parte dos resultados, uma vez que a pesquisa holandesa foi conduzida na população geral, enquanto que o ELSA-Brasil se confina a uma coorte de servidores públicos. Por esse motivo também, as análises apresentadas podem não ser generalizáveis para toda a população brasileira, já que o domínio de estudo se restringe a um estrato populacional que, em geral, tem maior nível de escolaridade e maior acesso ao capital social do que o conjunto dos brasileiros. Ainda assim, é possível que os resultados se apliquem às camadas médias urbanas que têm emprego. De toda a sorte, seria proveitoso aplicar o RG a heterogêneos grupos populacionais visando, assim, a alargar a avaliação da estabilidade (invariância) de sua estrutura fatorial 31,39.

Como visto, alguns refinamentos analíticos merecem enfrentamento em estudos futuros, como por exemplo, investigar a aludida existência de um fator de ordem superior ou de inva- riância métrica e escalar em diferentes domínios populacionais. Em particular, essas avaliações poderiam ser estendidas a análises de outros subgrupos, estratificando-se por faixa etária, sexo ou nível de escolaridade. Ademais, uma ampla avaliação da validade de constructo é recomendável, buscando-se examinar as relações entre as dimensões identificadas na análise fatorial e outros constructos teóricos conexos apontados na literatura. Por fim, seria interessante avaliar a validade preditiva do capital social em relação a diferentes desfechos de saúde, uma proposta viável no contexto de estudos longitudinais como o ELSA-Brasil. No conjunto, essas abordagens poderão complementar o escrutínio das propriedades psicométricas do RG, no sentido de reforçar a adequabilidade de seu uso no contexto brasileiro.

Em síntese, a avaliação da estrutura fatorial do instrumento permitiu identificar uma versão composta por 25 itens distribuídos em três dimensões, mas sendo uma delas mapeada por apenas dois itens. Assim sendo, a utilização das duas dimensões restantes merecem ser recomendadas em outros estudos brasileiros com populações semelhantes, seja considerando as escalas na sua forma bruta - por meio de escores totais formados pelo somatório dos escores de itens componentes -, seja como variáveis latentes - por meio de escores fatoriais obtidos via análises de modelos de equação estrutural.

\section{Colaboradores}

E. P. Souto, A. G. G. Vasconcelos, M. E. Reichenheim e R. H. Griep participou da concepção e desenho do estudo, análise e interpretação dos dados e redação de todas as versões do artigo. D. Chor participou da revisão crítica do artigo e da redação de sua versão final.

\section{Agradecimentos}

Os autores agradecem aos participantes e à equipe de pesquisa do ELSA-Brasil. 


\section{Referências}

1. Lin N. Social capital: a theory of social structure and action. Cambridge: Cambridge University Press; 2001.

2. Bourdieu P. The forms of capital. In: Richardson JE, editor. Handbook of theory and research for the sociology of education. New York: Greenwood Press; 1986. p. 47-58.

3. Putnam RD. Comunidade e democracia: a experiência da Itália moderna. Rio de Janeiro: Editora FGV; 2002.

4. Coleman JS. Social capital in the creation of human capital. AJS 1988; 94 Suppl:S95-S120.

5. Webber MP, Huxley PJ. Measuring access to social capital: the validity and reliability of the Resouce Generator-UK and its association with common mental disorder. Soc Sci Med 2007; 65:481-92.

6. Fujiwara T, Kawachi I. A prospective study of individual-level social capital and major depression in the United States. J Epidemiol Community Health 2008; 62:627-33.

7. Aslund C, Starrin B, Nilsson KW. Social capital in relation to depression, musculoskeletal pain, and psychosomatic symptoms: a cross-sectional study of a large population-based cohort of Swedish adolescents. BMC Public Health 2010; 10:715.

8. Ramlagan S, Peltzer K, Phaswana-Mafuya N. Social capital and health among older adults in South Africa. BMC Geriatr 2013; 13:100.

9. Giordano GN, Jonas B, Lindström M. Social capital and self-rated health - a study of temporal (causal) relationships. Soc Sci Med 2012; 75:340-8.

10. Chola L, Alaba O. Association of neighbourhood and individual social capital, neighbourhood economic deprivation and self-rated health in South Africa - a Multi-Level Analysis. PLoS One 2013; 8:e71085.

11. Kobayashi T, Kawachi I, Iwase T, Suzuki E, Takao S. Individual-level social capital and self-rated health in Japan: an application of the Resource Generator. Soc Sci Med 2013; 85:32-7.

12. Langille DB, Asbridge M, Kisely S, Rasic D. Suicidal behaviours in adolescents in Nova Scotia, Canada: protective associations with measures of social capital. Soc Psychiatry Psychiatr Epidemiol 2012; 47:1549-55.

13. Smith N, Kawachi I. State-level social capital and suicide mortality in the 50 U.S. states. Soc Sci Med 2014; 120:269-77.

14. Ueshima K, Fujiwara T, Takao S, Suzuki E, Iwase T, Doi $\mathrm{H}$, et al. Does social capital promote physical activity? A population-based study in Japan. PLoS One 2010; 5:e12135.

15. Kouvonen A, Oksanen T, Vahtera J, Väänänen A, Roberto DV, Elovainio M, et al. Work-place social capital and smoking cessation: the Finnish Public Sector Study. Addiction 2008; 103:1857-65.

16. Moore S, Daniel M, Paquet C, Dubé L, Gauvin L. Association of individual network social capital with abdominal adiposity, overweight and obesity. J Public Health (Oxf) 2009; 31:175-83.
17. Farajzadegan Z, Jafari N, Nazer S, Keyvanara M, Zamani A. Social capital - a neglected issue in diabetes control: a cross-sectional survey in Iran. Health Soc Care Community 2013; 21:98-103.

18. Portes A. Social capital: its origins and applications in modern sociology. Annu Rev Sociol 1998; 24: $1-24$

19. Lin N, Fu Y, Hsung R. Position generator: a measurement for social capital. In: Lin N, Cook R, Burt R, editors. Social capital: theory and research. New York: Cambridge University Press; 1998. p. 51-87.

20. van der Gaag MPJ. Measurement of individual social capital [Doctoral Dissertation]. Groningen: Universidade de Groningen; 2005.

21. Agampodi TC, Agampodi SB, Glozier N, Siribaddana S. Measurement of social capital in relation to health in low and middle income countries (LMIC): a systematic review. Soc Sci Med 2015; 128:95-104.

22. van der Gaag MPJ, Snijders TAB. The resource generator: measuring social capital with concrete items. Soc Networks 2005; 27:1-29.

23. Lannoo S. Social capital from an Individual Perspective. An investigation into the influence of urbanization and the tenure of private resources on the social resources of individuals. In: Proceedings of the Conference the Social Differentiation of Trust and Social Capital. https://biblio.ugent.be/ publication/764570 (acessado em 20/Mar/2015).

24. Grinstein-Weiss M, Yeo YH, Manturuk KR, Despard MR, Holub KA, Greeson JKP, et al. Social capital and homeownership in low to moderate-income neighborhoods. Social Work Research 2013; 37:3753

25. Häuberer J. Social capital theory: towards a methodological foundation. Prague: VS Research; 2011.

26. Griep RH, Chor D, Santos SM, Fonseca MJM, Cardoso LO, Alves MGM, et al. Capital social no ELSABrasil: confiabilidade teste-reteste do Resource Generator scale. Rev Saúde Pública 2013; 47 Suppl 2:131-9.

27. Aquino E, Barreto S, Bensenor I, Carvalho M, Chor D, Duncan B, et al. Brazilian Longitudinal Study of Adult Health (ELSA-Brasil): objectives and design. Am J Epidemiol 2012; 175:315-24.

28. Chor D, Alves MGM, Giatti L, Cade NV, Nunes MA, Molina MCB, et al. Questionário do ELSA-BRASIL: desafios na elaboração de instrumento multidimensional. Rev Saúde Pública 2013; 47 Suppl 2: 27-36.

29. Schmidt MI, Griep RH, Passos VM, Luft VC, Goulart AC, Menezes GMdS, et al. Estratégias e desenvolvimento de garantia e controle de qualidade no ELSA-Brasil. Rev Saúde Pública 2013: 47 Suppl 2: 105-12.

30. Marsh H, Muthén B, Asparouhouv A, Morin A, Lüdtke O, Robitzsch A, et al. Exploratory structural equation modeling, integrating CFA and EFA: application to students' evaluations of university teaching. Struct Equ Modeling 2009; 16:439-76. 
31. Brown TA. Confirmatory factor analysis for applied reaserch. 2nd Ed. New York: Guilford Press; 2015.

32. Hair JF, Black WC, Babin BJ, Anderson RE, Tatham RL. Análise multivariada de dados. 6a Ed. Porto Alegre: Bookman; 2009.

33. Raykov T, Shrout P. Reliability of scales with general structure: point and interval estimation using a structural equation modeling approach. Struct Equ Modeling 2002; 9:195-212.

34. Fornell C, Larcker DF. Evaluating structural equation models with unobservable variables and measurement error. J Mark Res 1981; 18:39-50.

35. Kline RB. Principles and practice of structural equation modeling. New York: Guilford Press; 2011.

\begin{abstract}
This study aims to analyze the factor structure of the Brazilian version of the Resource Generator (RG) scale, using baseline data from the Brazilian Longitudinal Health Study in Adults (ELSA-Brasil). Cross-validation was performed in three random subsamples. Exploratory factor analysis using exploratory structural equation models was conducted in the first two subsamples to diagnose the factor structure, and confirmatory factor analysis was used in the third to corroborate the model defined by the exploratory analyses. Based on the 31 initial items, the model with the best fit included 25 items distributed across three dimensions. They all presented satisfactory convergent validity (values greater than 0.50 for the extracted variance) and precision (values greater than 0.70 for compound reliability). All factor correlations were below 0.85 , indicating full discriminative factor validity. The RG scale presents acceptable psychometric properties and can be used in populations with similar characteristics.
\end{abstract}

Surveys and Questionnaires; Social Capital; Validation Studies; Statistical Factor Analysis
36. Velicer WF, Fava JL. Effects of variable and subject sampling on factor pattern recovery. Psychol Methods 1998; 3:231-51.

37. Pett MA, Lackey NR, Sullivan JJ. Making sense of factor analysis: the use of factor analysis for instrument development in health care research. London: Sage Publications; 2003.

38. Floyd FJ, Widaman KF. Factor analysis in the development and refinement of clinical assessment instruments. Psychol Assess 1995; 7:286-99.

39. Kankaras M, Vermunt J, Moors G. Measurement equivalence of ordinal items: a comparison of factor analytic, item response theory and latent class approaches. Sociol Methods Res 2011; 40:279-310.

\section{Resumen}

Esta investigación tiene por objetivo estudiar la estructura factorial de la versión brasileña de la escala Resource Generator (RG), utilizando datos de la línea de base del Estudo Longitudinal de Saúde do Adulto (ELSA-Brasil). Se realizó una prueba de validación cruzada con tres sub-muestras aleatorias. El análisis factorial exploratorio, usando modelos de ecuación estructural exploratorio, fue realizado en las dos primeras sub-muestras para diagnóstico de la estructura factorial y, en la tercera, el análisis factorial confirmatorio fue utilizado para corroborar el modelo definido por los análisis exploratorios. A partir de los 31 items iniciales, el modelo de mejor ajuste contempló 25 ítems distribuidos en tres dimensiones. Todos presentaron validez convergente (valores por encima de 0,50 para la variancia extraída) y precisión (valores por encima de 0,70 para la confiabilidad compuesta) satisfactorias. Todas las correlaciones factoriales estuvieron por debajo de 0,85 , indicando plena validez factorial discriminante. La escala RG presenta propiedades psicométricas aceptables y puede ser utilizada en poblaciones con características semejantes.

Encuestas y Cuestionarios; Capital Social; Estudios de Validación; Análisis Factorial
Recebido em 26/Jun/2015

Versão final reapresentada em 09/Fev/2016 Aprovado em 11/Mar/2016 